\title{
Where do small, weakly inertial particles go in a turbulent flow?
}

\author{
Mathieu Gibert ${ }^{1,4} \ddagger$, Haitao $\mathbf{X u}^{1,4}$ and Eberhard Bodenschatz ${ }^{1,2,3,4} \dagger$ \\ ${ }^{1}$ Max Planck Institute for Dynamics and Self Organization (MPIDS), 37077 Göttingen, Germany \\ 2 Institute for Nonlinear Dynamics, University of Göttingen, 37077 Göttingen, Germany \\ ${ }^{3}$ Laboratory of Atomic and Solid-State Physics and Sibley School of Mechanical and Aerospace \\ Engineering, Cornell University, Ithaca, NY 14853, USA \\ ${ }^{4}$ International Collaboration for Turbulence Research
}

(Received 5 December 2011; revised 25 January 2012; accepted 3 February 2012; first published online 27 March 2012)

We report experimental results on the dynamics of heavy particles of the size of the Kolmogorov scale in a fully developed turbulent flow. The mixed Eulerian structure function of two-particle velocity and acceleration difference vectors $\left\langle\delta_{r} \boldsymbol{v} \cdot \delta_{r} \boldsymbol{a}_{p}\right\rangle$ was observed to increase significantly with particle inertia for identical flow conditions. We show that this increase is related to a preferential alignment between these dynamical quantities. With increasing particle density the probability for those two vectors to be collinear was observed to grow. We show that these results are consistent with the preferential sampling of strain-dominated regions by inertial particles.

Key words: homogeneous turbulence, isotropic turbulence, particle/fluid flows

\section{Introduction}

Turbulence occurs whenever fluid viscous forces are small compared with the dominant driving forces of the flow. In practice, this includes most macroscopic natural and technological flows. Very frequently, these turbulent flows are loaded with small, passive particles with non-negligible inertia, i.e. particles do not exactly follow fluid motion. Familiar examples of such flows include clouds in the atmosphere, sand storms in arid regions, flow-driven transports of grains in pharmaceutical and food industries, fluidized beds in chemical engineering and fuel sprays in combustion engines and turbines. The understanding of particle-turbulence interaction is therefore of central importance to our ability to make advances in key economical and societal issues such as energy generation, climate change and pollution control. Compared with the fluid turbulence problem itself, which is well described by the nonlinear Navier-Stokes equations, the particle-turbulence interaction problem is on much weaker foundations. In principle, the particle dynamics can be obtained by solving particle equations coupled with the Navier-Stokes equations for the fluid with no-slip boundary conditions on particle surfaces. However, this approach is a formidable task for theoretical and numerical investigations (Happel \& Brenner 1965; Homann \& Bec 2010). Most theoretical analyses and numerical simulations therefore

$\dagger$ Email address for correspondence: eberhard.bodenschatz@ds.mpg.de $\ddagger$ Present address: Institut NÉEL CNRS/UJF (Grenoble France). 
must rely on simplified equations, in which the particle-turbulence interactions are modelled. An interesting result obtained from these simplified equations is that in a turbulent flow particles with small to moderate inertia and sizes smaller than the Kolomogrov length scale tend to move to regions with high strain and low vorticity (Maxey \& Riley 1983). This so-called 'preferential concentration' of inertial particles is under intensive investigation (Sundaram \& Collins 1997; Collins \& Keswani 2004; Falkovich \& Pumir 2004; Chun et al. 2005; Ducasse \& Pumir 2008; Zaichik \& Alipchenkov 2009) and has been invoked when studying droplet collision rates in warm clouds (Falkovich, Fouxon \& Stepanov 2002; Shaw 2003) and plankton encounter rates in the ocean (Schmitt \& Seuront 2008). Inhomogeneous distributions of inertial particles in turbulent flows have also been observed in several experiments (Aliseda et al. 2002; Wood, Hwang \& Eaton 2005; Salazar et al. 2008; Saw et al. 2008; Monchaux, Bourgoin \& Cartellier 2010). Here we show direct experimental evidence that regions of high particle concentrations are also regions of high strain.

\section{Experimental method and observation}

We conducted three-dimensional Lagrangian particle-tracking measurements (Ouellette, $\mathrm{Xu} \&$ Bodenschatz 2006; Xu 2008) and obtained the trajectories of solid particles with different densities in a turbulent water flow. Below we give a brief summary of the experiment (see also Gibert, Xu \& Bodenschatz 2010). The turbulent flow was generated by two counter-rotating baffled discs of $28 \mathrm{~cm}$ in diameter. The turbulence chamber, shaped as a hexagonal cylinder, measured $40 \mathrm{~cm}$ along the axis of the propellers and $38 \mathrm{~cm}$ in the cross-section, both in height (vertically) and width (horizontally). The axis of rotation of the discs was in the horizontal direction. The strong sweeping of the fluid near the bottom surface resuspended the heavy particles with the effect that the particle concentration remained sufficient for the measurement of multi-particle statistics. A spherically shaped measurement volume with a diameter of $22 \mathrm{~mm}$ was located at the centre of the water chamber, where the mean flow was small compared with the velocity fluctuations. The spatial resolution of $45 \mu \mathrm{m}$ per pixel and the temporal resolution of 31 frames per Kolmogorov time $\tau_{\eta}$ guaranteed accurate measurements of particle velocity and acceleration ( $\mathrm{Xu}$ et al. 2007). As shown in the table 1 we used three different types of particles: polystyrene, glass and stainless steel with densities between $1 \lesssim \rho_{p} / \rho_{f} \lesssim 8$. For the Taylor microscale Reynolds number of the turbulent flow $\left(R_{\lambda}=442\right)$, the particles had the size of the Kolmogorov scale $(\eta=72 \mu \mathrm{m})$. The resulting Stokes numbers, a relative measure of the particle inertia, were between 0.08 and 0.5 (see table 1). Other relevant parameters of the turbulent flow are the fluctuating velocity $u^{\prime}=0.15 \mathrm{~m} \mathrm{~s}^{-1}$, the integral length scale $L=87 \mathrm{~mm}$ and the Kolmogorov time scale $\tau_{\eta}=5.2 \mathrm{~ms}$. We measured $3 \times 10^{7}$ data points for the polystyrene particles, $8 \times 10^{7}$ for the glass particles and $3 \times 10^{6}$ for the steel particles. The fluctuations in figure 1 can be attributed to the statistical uncertainty.

For these particles, we observed that the magnitude of the mixed velocity-acceleration Eulerian structure function $\left\langle\delta_{r} \boldsymbol{v} \cdot \delta_{r} \boldsymbol{a}_{\boldsymbol{p}}\right\rangle$ increased with particle density, where $\delta_{r} \boldsymbol{v}$ and $\delta_{r} \boldsymbol{a}_{\boldsymbol{p}}$ are the relative velocity and the relative acceleration between two particles separated at distance $r$ (e.g. $\left.\delta_{r} \boldsymbol{v}=\boldsymbol{v}(x+r, t)-\boldsymbol{v}(x, t)\right)$. For fluid tracers this mixed velocity-acceleration structure function in the inertial range equals a constant: $\left\langle\delta_{r} \boldsymbol{u} \cdot \delta_{r} \boldsymbol{a}\right\rangle=-2 \epsilon$, where $\boldsymbol{u}$ and $\boldsymbol{a}$ are fluid velocity and acceleration, 


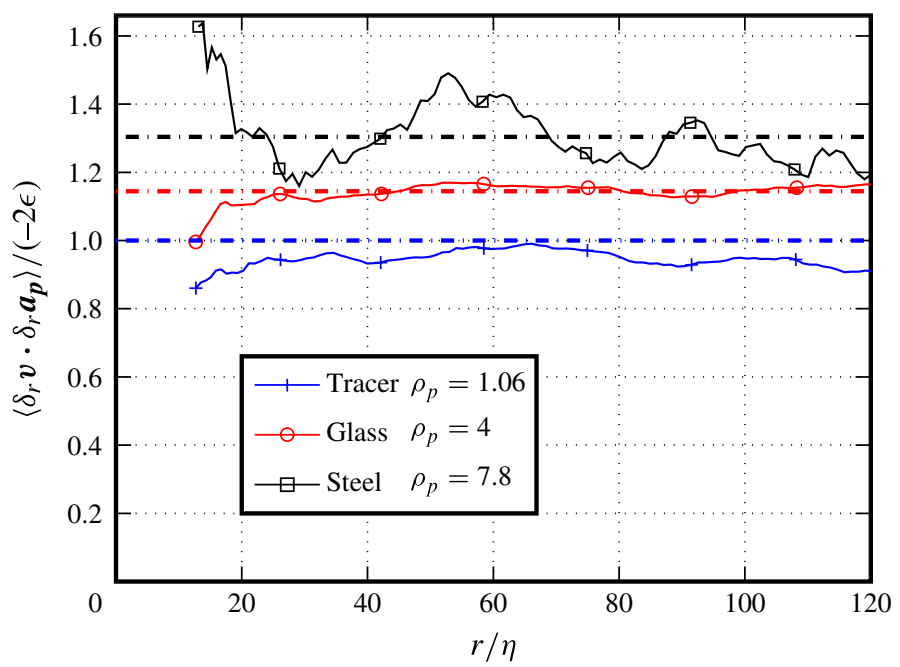

FIGURE 1. (Colour online available at journals.cambridge.org/flm) Mixed Eulerian velocity-acceleration structure function $\left\langle\delta_{r} \boldsymbol{v} \cdot \delta_{r} a_{p}\right\rangle$ normalized by the expected values for the fluid particles $-2 \epsilon$. The values of the different plateaus represented by the dot-dashed lines are: blue 1 , red 1.15 , black 1.30 .

$\begin{array}{lcccc} & \rho_{p} / \rho_{f} & d_{p}(\mu \mathrm{m}) & d_{p} / \eta & S t \\ \text { Polystyrene } & 1.06 & 74 \pm 10 & 0.98 & 0.09 \\ \text { Glass } & 4 & 75 \pm 8 & 1.04 & 0.27 \\ \text { Steel } & 7.8 & 75 \pm 15 & 1.04 & 0.50\end{array}$

TABLE 1. Characteristics of the different particles used in the experiments. The Stokes number is defined as $S t \equiv d_{p}^{2} / 12 \beta \eta^{2}$, where $d_{p}$ is the particle diameter, $\beta \equiv 3 \rho_{f} /\left(2 \rho_{p}+\rho_{f}\right)$ is the modified density ratio, that takes into account the added mass effect, and $\eta$ is the Kolmogorov length scale of the turbulence.

respectively, and $\epsilon$ is energy dissipation rate per unit mass (Mann, Ott \& Andersen 1999; Falkovich, Gawędzki \& Vergassola 2001; Pumir, Shraiman \& Chertkov 2001; Hill 2006). We also found this in our experiment, where the polystyrene particles behaved as tracers (Gibert et al. 2010). As shown in figure 1, for inertial particles, this mixed velocity-acceleration structure functions in the inertial range were still constant, but the values increased significantly (up to $30 \%$ ) when the particle Stokes number increased from 0.08 to 0.44 .

As reported earlier (Gibert et al. 2010) the relative velocities in this range changed by less than $10 \%$. In addition, the root-mean-square (r.m.s.) acceleration changed with particle density from $6.9,6.6$ and $8.5 \mathrm{~m} \mathrm{~s}^{-2}$ for tracer, glass and steel particles, respectively. Therefore, the observed change in $\left\langle\delta_{r} \boldsymbol{v} \cdot \delta_{r} \boldsymbol{a}_{p}\right\rangle$ cannot be simply explained by changes in the magnitudes of $\delta_{r} \boldsymbol{v}$ and $\delta_{r} \boldsymbol{a}_{p}$, but must be associated with the alignment between the two vectors. Indeed, we observed a change of the cosines

$$
\cos \theta=\frac{\delta_{r} \boldsymbol{v} \cdot \delta_{r} \boldsymbol{a}_{\boldsymbol{p}}}{\left|\delta_{r} \boldsymbol{v}\right|\left|\delta_{r} \boldsymbol{a}_{\boldsymbol{p}}\right|}
$$



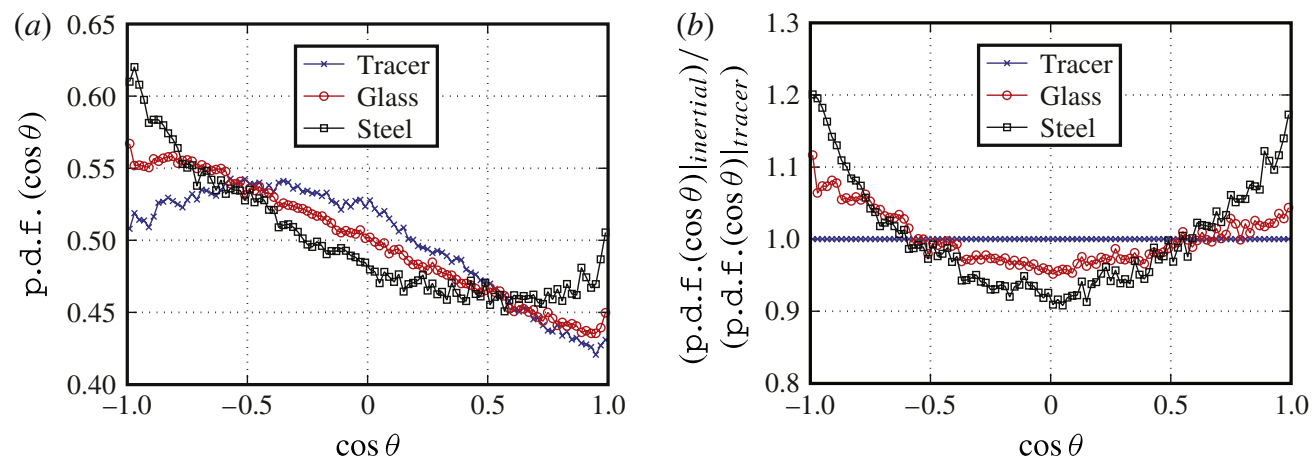

FIgURE 2. (Colour online) (a) The p.d.f. of the cosine of the angle $\theta$ between $\delta_{r} \boldsymbol{v}$ and $\delta_{r} \boldsymbol{a}_{p}$ for the three types of particles for a separation $r / \eta=28 \pm 7$. (b) Same p.d.f.s as in $(a)$ normalized by the p.d.f. of the tracer particles; $(b)$ shows the relative effect of the particle inertia on the alignment/anti-alignment of $\delta_{r} \boldsymbol{v}$ with $\delta_{r} \boldsymbol{a}_{p}$.

of the angle between these two vectors with inertia of the particles. Considering the tracer particles (polystyrene), its average value $\langle\cos \theta\rangle$ monotonically increases from -0.052 to -0.01 when increasing the observation scale $r(12<r / \eta<120)$. For glass and steel particles, the average $\langle\cos \theta\rangle$ increased in absolute value by $25 \%$ and $9 \%$, respectively.

\section{Alignment of relative acceleration and relative velocity}

The effect of particle inertia on $\cos \theta$ is best demonstrated by its probability density function (p.d.f.). Figure 2(a) shows the p.d.f.s of $\cos \theta$ corresponding to $\delta_{r} \boldsymbol{v}$ and $\delta_{r} \boldsymbol{a}_{\boldsymbol{p}}$ at separation $r=(28 \pm 7) \eta$ for the three different types of particles (similar behaviour is observed for other separations in the inertial range). All p.d.f.s are skewed to the side of $\cos \theta<0$, indicating that the two vectors are preferentially oriented in opposite directions, which gives the negative values of $\left\langle\delta_{r} \boldsymbol{v} \cdot \delta_{r} \boldsymbol{a}_{p}\right\rangle$, as shown in figure 1 .

A very interesting observation from figure $2(a)$ is the increase of the probability of $|\cos \theta| \approx 1$ with particle inertia. This can be seen even better in figure $2(b)$ where the p.d.f.s of the two types of inertial particles were normalized by the p.d.f. of the fluid tracers. Clearly, the particle inertia enhanced the collinearity, either in the same direction or in the opposite direction, between the relative velocity and the relative acceleration vectors. We note that the p.d.f.s are slightly skewed toward the anti-alignment side $(\cos \theta<0)$.

How can we understand this observation? For this range of Stokes numbers, as shown numerically and experimentally (Bec et al. 2006; Gibert et al. 2010; Salazar $\&$ Collins 2012), the difference between particle and fluid velocity and acceleration at the particle position is very small. The same is true for the dynamical variables $\delta_{r} \boldsymbol{a}_{p}$ and $\delta_{r} \boldsymbol{v}$. Thus, if the inertial particles were homogeneously distributed in space, like fluid tracers, we should not observe any differences between the p.d.f.s of $\cos \theta$ for fluid tracers and inertial particles. This is not what we observed. Therefore, our measurements strongly indicate that inertial particles preferentially explore certain regions of the flow, leading to the observed p.d.f.s in figure 2.

\section{Implication to preferential sampling}

To further elucidate which features of the turbulence are responsible for the observed behaviour, we investigate $\delta_{r} \boldsymbol{u}$ and $\delta_{r} \boldsymbol{a}$ of fluid tracers for simple flow fields. 
Let us consider a simple model, namely a steady, incompressible linear velocity field $u_{i}$ defined by

$$
u_{i}=M_{i j} x_{j}
$$

where $u_{i}$ is the fluid velocity at position $x_{i}, M_{i j}$ is the velocity gradient tensor. In our experiments, where the separation $r$ are in the inertial range, we could treat $M_{i j}$ as an effective velocity gradient at scale $r$. It has been shown that the effective velocity gradient at scale $r$ evolves with a time scale $t_{0} \equiv\left(r^{2} / \epsilon\right)^{1 / 3}$ (Xu, Pumir \& Bodenschatz 2011, and authors' unpublished observations), which is much larger than the particle relaxation time $\tau_{p}$ for $r$ in the inertial range, as it can be quantified by noticing that the ratio $\tau_{p} / t_{0}=S t(\eta / r)^{2 / 3} \ll 1$. Therefore, to study the dynamics of particle relative velocity and relative acceleration, the flow field may be treated as frozen.

For a steady flow, the fluid acceleration field $a_{i}$ is determined by $M_{i j}$ : $a_{i}=u_{j} \partial u_{i} / \partial x_{j}=M_{i k} M_{k j} x_{j}$. The expressions for two-particle relative velocity and acceleration, together with the cosine of the angle between them, $\cos \theta$, can then be obtained in closed form. For a velocity field with pure rotation, $M_{i j}=-M_{j i}$, it can be easily shown that $\delta_{r} \boldsymbol{a} \cdot \delta_{r} \boldsymbol{u}=0$, i.e. the fluid acceleration is always normal to its velocity. The p.d.f. of $\cos \theta$ is a $\delta$ function at $\cos \theta=0$. This is not consistent with our observations.

For a purely straining velocity field, the three eigenvalues of $M_{i j}$ are all real and can be arranged as $\lambda_{1} \geqslant \lambda_{2} \geqslant \lambda_{3}$. Incompressibility requires that $\lambda_{1}+\lambda_{2}+\lambda_{3}=0$. Then it is straightforward to show that the alignment between relative velocity and relative acceleration vectors depends only on the orientation of the separation vector $\boldsymbol{r}$ between the two particles and the ratio of the eigenvalues, e.g. $\lambda_{2} / \lambda_{1}$. The p.d.f. of $\cos \theta$ at a given $\lambda_{2} / \lambda_{1}$ can then be obtained by assuming that $\boldsymbol{r}$ distributes uniformly in all directions within this purely straining flow. When $\lambda_{2}=0$, i.e. the two-dimensional case, the p.d.f. is

$$
\text { p.d.f. }(\cos \theta)=\frac{1}{\pi\left(1-\cos ^{2} \theta\right)^{1 / 2}} .
$$

For more general cases, $\lambda_{2} / \lambda_{1} \neq 0$, we obtained the p.d.f.s numerically by choosing randomly $10^{7}$ separation vectors $\boldsymbol{r}$ and computing $\cos \theta((2.1))$ in the linear velocity field $u_{i}((4.1))$. Please note, numerical analysis of various test cases showed that the shape of the p.d.f. was insensitive to specifics of the particle distribution. It is well known that in turbulent flows the average of the eigenvalue ratio of the velocity gradient is positive, with a value of $\left\langle\lambda_{2} / \lambda_{1}\right\rangle \approx 0.2$ (Betchov 1956; Ashurst et al. 1987; Kholmyansky, Tsinober \& Yorish 2001; Lüthi, Tsinober \& Kinzelbach 2005; Berg et al. 2006). For the effective velocity gradient in the inertial range, this ratio decreases monotonically from this positive value to 0 when the scale $r$ increases from $\eta$ to the integral scale (authors' unpublished observations). We therefore performed simulations with the range of $\lambda_{2} / \lambda_{1}$ around these values. Figure 3 shows the p.d.f.s of $\cos \theta$ from the simulation results with $0 \leqslant \lambda_{2} / \lambda_{1} \leqslant 0.3$, together with the analytical solution equation (4.2). The p.d.f.s for $\lambda_{2} / \lambda_{1}>0$ in figure 3 are remarkably similar to the 'relative p.d.f.s' shown in figure $2(b)$, namely, the relative accelerations and the relative velocities prefer to be collinear, with slight skewness to the anti-alignment side. This observation, together with the fact that inertial particles have, to leading order, the velocities and accelerations of the fluid at the particle positions (Bec et al. 2006; Gibert et al. 2010; Salazar \& Collins 2012), suggests that inertial particles sample preferentially strain-dominated regions in turbulence. Our approach should 


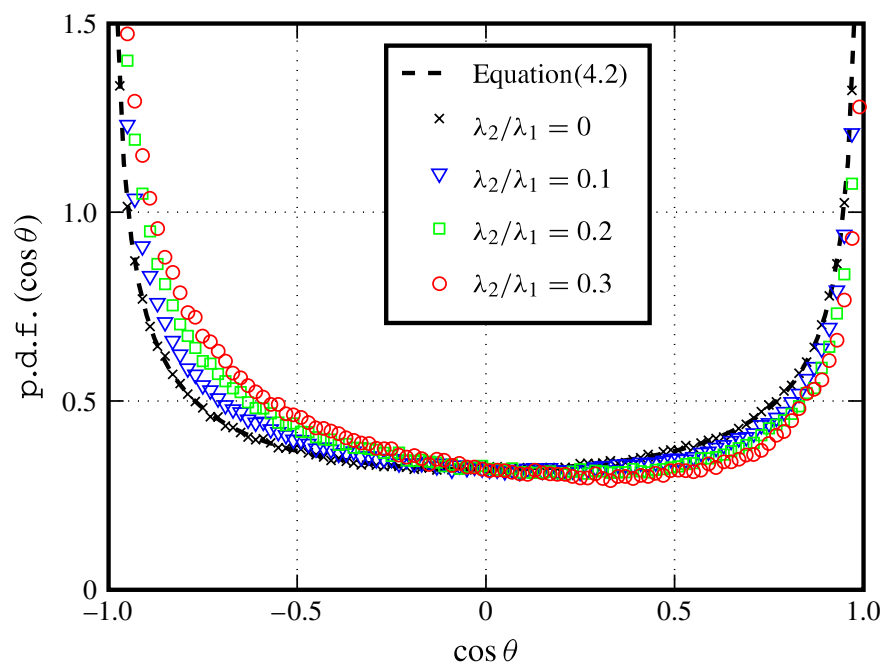

FIGURE 3. (Colour online) The p.d.f. of $\cos \theta$ as defined in (2.1). The relative velocity and acceleration are obtained from two fluid tracers randomly distributed in a steady, purely straining linear velocity field. The different symbols corresponds to different ratios of $\lambda_{2} / \lambda_{1}$. The dashed line corresponds to (4.2).

be compared with results from numerical simulations of simplified equations, for which the phenomenon of 'preferential sampling of strain-dominated regions' has been observed (Collins \& Keswani 2004; Chun et al. 2005). There the velocity fields around the virtual inertial point particles were investigated. It will be interesting to see how well the p.d.f.s of $\cos \theta$ agree with the experimental results presented here. Moreover, studying the p.d.f. of $\cos \theta$ for fluid tracers might reveal new information about the structures of the turbulent flow itself, e.g. by conditioning of the p.d.f. on local strain rates and vorticities.

\section{Summary}

In summary, we observed a significant increase of the mixed velocity-acceleration structure function $\left\langle\delta_{r} \boldsymbol{v} \cdot \delta_{r} \boldsymbol{a}_{p}\right\rangle$ when increasing the inertia of heavy particles of the size of the Kolmogorov-scale. We found that this increase could not be attributed to the change in the magnitudes of the velocity and/or acceleration increments $\left(\left|\delta_{r} \boldsymbol{v}\right|\right.$ and/or $\left.\left|\delta_{r} \boldsymbol{a}_{p}\right|\right)$. A significant contribution to this effect is from the alignment or anti-alignment between the relative velocity and relative acceleration. Using a simple model, i.e. a linear velocity field, we show that in the inertial range heavy particles preferentially explore strain-dominated regions of a turbulent flow.

The tendency of heavy particles to preferentially explore strain-dominated regions may also explain our recent observations ( $\mathrm{Xu} \&$ Bodenschatz 2008) that the timeaveraged radial distribution function of particle positions increases when the centre of the von Kármán flow is approached. In that case, the long-time-averaged mean flow imposes deterministic strain at the centre of the apparatus. With our new findings reported here, one would expect that the long-time average would lead to a measurable preferential concentration towards the centre of the apparatus.

Our analysis does not rely on the commonly used and hard to measure long time average of the relative particle positions. Instead it only considers dynamical properties 
and thus opens a novel way to study particle turbulence interaction. For higher Stokes number, the filtering induced by the particle response time (Bec et al. 2006; Salazar \& Collins 2012) will have an additional effect on the analysis presented above.

\section{Acknowledgements}

We are grateful to R. J. Hill, A. Pumir and E.-W. Saw for many interesting discussions. Support from COST Action MP0806 is kindly acknowledged. This work was funded by the Max Planck Society, and the Marie Curie Fellowship, Programme PEOPLE - Call FP7-PEOPLE-IEF-2008 Proposal No 237521.

\section{REFERENCES}

Aliseda, A., Cartellier, A., Hainaux, F. \& Lasheras, J. C. 2002 Effect of preferential concentration on the settling velocity of heavy particles in homogeneous isotropic turbulence. J. Fluid Mech. 468, 77-105.

Ashurst, W. T., Kerstein, A. R., Kerr, R. M. \& Gibson, C. H. 1987 Alignment of vorticity and scalar gradient with strain rate in simulated Navier-Stokes turbulence. Phys. Fluids 30 (8), 2343-2353.

Bec, J., Biferale, L., Boffetta, G., Celani, A., Cencini, M., Lanotte, A., Musacchio, S. \& TosCHI, F. 2006 Acceleration statistics of heavy particles in turbulence. J. Fluid Mech. 550, 349-358.

Berg, J., LÜthi, B., MANN, J. \& OTt, S. 2006 Backwards and forwards relative dispersion in turbulent flow: An experimental investigation. Phys. Rev. E 74, 16304.

BetchOV, R. 1956 An inequality concerning the production of vorticity in isotropic turbulence. J. Fluid Mech. 1 (5), 497-504.

Chun, J. H., Koch, D. L., Rani, S. L., Ahluwalia, A. \& Collins, L. R. 2005 Clustering of aerosol particles in isotropic turbulence. J. Fluid Mech. 536, 219-251.

Collins, L. R. \& KesWANI, A. 2004 Reynolds number scaling of particle clustering in turbulent aerosols. New J. Phys. 6, 119.

Ducasse, L. \& Pumir, A. 2008 Intermittent particle distribution in synthetic free-surface turbulent flows. Phys. Rev. E 77 (6), 066304.

Falkovich, G., Fouxon, A. \& Stepanov, M. G. 2002 Acceleration of rain initiation by cloud turbulence. Nature 419, 151-154.

Falkovich, G., Gawȩdzki, K. \& Vergassola, M. 2001 Particles and fields in fluid turbulence. Rev. Mod. Phys. 73, 913.

FAlKovich, G. \& PUMiR, A. 2004 Intermittent distribution of heavy particles in a turbulent flow. Phys. Fluids 16 (7), L47-L50.

Gibert, M., Xu, H. \& Bodenschatz, E. 2010 Inertial effects on two-particle separation in a turbulent flows. Europhys. Lett. 90 (6), 64005.

HAppel, J. \& Brenner, H. 1965 Low Reynolds Number Hydrodynamics. Prentice-Hall.

HILL, R. J. 2006 Opportunities for use of exact statistical equations. J. Turbul. 7 (43), 1-13.

Homann, H. \& BEC, J. 2010 Finite-size effects in the dynamics of neutrally buoyant particles in turbulent flow. J. Fluid Mech. 651, 81-91.

Kholmyansky, M., Tsinober, A. \& Yorish, S. 2001 Velocity derivatives in the atmospheric surface layer at $R e_{\lambda}=10^{4}$. Phys. Fluids 13, 311 .

LÜthi, B., Tsinober, A. \& KinZelbaCh, W. 2005 Lagrangian measurement of vorticity dynamics in turbulent flow. J. Fluid Mech. 528, 87.

MAnN, J., Ott, S. \& Andersen, J. S. 1999 Experimental study of relative, turbulent diffusion. Ris $\phi-R-1036(E N)$.

MAXEY, M. \& RILEY, J. 1983 Equation of motion for a small rigid sphere in a nonuniform flow. Phys. Fluids 26, 883-889.

Monchaux, R., Bourgoin, M. \& Cartellier, A. 2010 Preferential concentration of heavy particles: A Voronoï analysis. Phys. Fluids 22, 103304. 
Ouellette, N. T., XU, H. \& Bodenschatz, E. 2006 A quantitative study of three-dimensional Lagrangian particle tracking algorithms. Exp. Fluids 40, 301-313.

Pumir, A., Shraiman, B. \& Chertkov, M. 2001 The Lagrangian view of energy transfer in turbulent flow. Europhys. Lett. 56 (3), 379-385.

Pumir, A., XU, H. \& Bodenschatz, E. 2012 Tetrad deformation and alignment of vorticity and strain in a turbulent flow (in preparation).

SAlazar, J. P. L. C \& Collins, L. R 2012 Inertial particle relative velocity statistics in homogeneous isotropic turbulence. J. Fluid Mech. 696, 45-67.

Salazar, J. P. L. C, Jong, J. D., CaO, L., Woodward, S. H., Meng, H. \& Collins, L. R. 2008 Experimental and numerical investigation of inertial particle clustering in isotropic turbulence. J. Fluid Mech. 600, 245-256.

Saw, E. W., Shaw, R. A., Ayyalasomayajula, S., Chuang, P. Y. \& Gylfason, A. 2008 Inertial clustering of particles in high-Reynolds-number turbulence. Phys. Rev. Lett. 100 (21), 214501.

SChMitt, F. C. \& SEURont, L. 2008 Intermittent turbulence and copepod dynamics: Increase in encounter rates through preferential concentration. J. Mar. Syst. 70, 263-272.

ShAw, R. A. 2003 Particle-turbulence interactions in atmospheric clouds. Annu. Rev. Fluid Mech. 35, $183-227$.

Sundaram, S. \& Collins, L. R. 1997 Collision statistics in an isotropic particle-laden turbulent suspension. 1. Direct numerical simulations. J. Fluid Mech. 335, 75-109.

Wood, A. M., Hwang, W. \& EATON, J. K. 2005 Preferential concentration of particles in homogeneous and isotropic turbulence. Intl J. Multiphase Flow 31 (10-11), 1220-1230.

XU, H. 2008 Tracking Lagrangian trajectories in position-velocity space. Meas. Sci. Technol. 19 (7), 075105 .

XU, H. \& Bodenschatz, E. 2008 Motion of inertial particles with size larger than Kolmogorov scale in turbulent flows. Physica D 237, 2095-2100.

Xu, H., Ouellette, N. T., Vincenzi, D. \& Bodenschatz, E. 2007 Acceleration correlations and pressure structure functions in high-Reynolds number turbulence. Phys. Rev. Lett. 99, 204501.

Xu, H., Pumir, A. \& Bodenschatz, E. 2011 The pirouette effect in turbulent flows. Nat. Phys. 7, 709-712.

ZAichiK, L. I. \& Alipchenkov, V. M. 2009 Statistical models for predicting pair dispersion and particle clustering in isotropic turbulence and their applications. New J. Phys. 11, 103018. 